\title{
Open-label clinical trial of alendronate after teriparatide therapy in people with spinal cord injury and low bone mineral density
}

\author{
Ifaz T. Haider ${ }^{1,2} \cdot$ Narina Simonian ${ }^{3,4} \cdot$ Amanpreet S. Saini $^{3} \cdot$ Frances M. Leung ${ }^{3} \cdot$ W. Brent Edwards ${ }^{1,2}$. \\ Thomas J. Schnitzer ${ }^{3}$
}

Received: 3 February 2019 / Revised: 6 May 2019 / Accepted: 7 May 2019 / Published online: 4 June 2019

(C) International Spinal Cord Society 2019

\begin{abstract}
Study design Non-randomized open-label clinical trial of oral alendronate after teriparatide therapy in people with spinal cord injury (SCI) and low bone mineral density (BMD).

Objectives To determine if alendronate would prevent bone loss after discontinuation of teriparatide.

Setting Outpatient research clinic at Northwestern University Feinberg School of Medicine.

Methods Seventeen participants with chronic SCI who recently completed 12-24 months of teriparatide treatment received oral alendronate $70 \mathrm{mg}$ once weekly for 12 months. Participants were evaluated at baseline, 6 months and 12 months. Bone was assessed by: DXA at the spine and hip, CT at the distal femur/proximal tibia, serum collected for bone markers, and bone strength determined by finite element (FE) analysis of the proximal tibia.

Results Areal BMD showed no significant change from baseline at the total hip or femoral neck, where mean change (SD) was $1.3 \%$ (4.7) and $0.54 \%$ (5.0), respectively. However, areal BMD increased significantly at the spine by $2.5 \%$ (4.6). CT demonstrated significant increases in bone mineral content at the femoral epiphysis, metaphysis, and diaphysis, 15\% (18), $7.7 \%$ (12), and 3.0\% (3.5), respectively. Measurements at the tibia illustrated improvements and reductions, but no changes to FE-predicted strength were observed. Biomarkers illustrated inhibition of bone formation and resorption, with P1NP and CTX decreasing by $52 \%$ (82) and $62 \%$ (74), respectively.

Conclusion Twelve months of alendronate after discontinuation of teriparatide in people with SCI can prevent bone loss and may increase bone mass and preserve bone strength at the spine, hip, and some sites of the knee.
\end{abstract}

\section{Introduction}

Many individuals with acute spinal cord injury (SCI) experience a significant reduction (up to 60\%) of bone mass and bone strength in their femur and tibia with bone loss

Thomas J. Schnitzer

tjs@northwestern.edu

1 Human Performance Laboratory, Faculty of Kinesiology, University of Calgary, Calgary, AB T2N 1N4, Canada

2 McCaig Institute for Bone and Joint Health, University of Calgary, Calgary, AB T2N 1N4, Canada

3 Department of Physical Medicine and Rehabilitation, Northwestern University Feinberg School of Medicine, Chicago, IL 60611, USA

4 Northwestern University Clinical and Translational Sciences Institute, Northwestern University Feinberg School of Medicine, Chicago, IL 60611, USA being most rapid in magnitude during the first 1-3 years after injury [1-3]; thereafter, the rate of bone loss slows, reaching a new steady state $[4,5]$. The rapid demineralization in the acute SCI setting stems primarily from the cessation of weight-bearing [6], which accompanies motor complete and many motor incomplete injuries [7]. By the time the injury reaches a chronic stage, the amount of bone mass remaining is low, making the bone weak and highly susceptible to fractures [8]. Fracture incidence in people with SCI increases with injury duration, with mean time to first fracture reported to be 6-9 years after SCI $[9,10]$. Fractures occur predominantly below the neurological level of the SCI lesion, most commonly occurring in the lower extremities, where bone loss and changes to bone microarchitecture are most significant $[9,11,12]$. These fractures are often a consequence of minimal to no trauma and can lead to secondary complications [12-14], further loss of independence, increased financial burden, and the likelihood of another fracture [15]. 
Currently, there is no standard of care to treat the existing bone loss and the high risk of fracture in the chronic SCI population. Rebuilding lost bone mass would be an important step in the reduction of fracture incidence. Therefore, treatment with anabolic agents, which increase bone strength and decrease fracture incidence by stimulating bone formation, would be the expected approach $[16,17]$. Our group has recently reported on the use of teriparatide [18], a peptide containing the biologically active N-terminal 34 amino acids of parathyroid hormone and the only anabolic bone agent available at the time the study was started, in people with chronic SCI. In that clinical trial, though treatment with teriparatide was not associated with robust improvements in bone mineral density (BMD) at the knee, treatment did result in modest increases in BMD at the spine and hip in people with long-standing SCI after 1 or 2 years of treatment.

Termination of teriparatide therapy without initiating further pharmacologic intervention is known to lead to rapid loss of the newly gained bone [19-22]. Treatment with alendronate after at least a year of teriparatide therapy has been demonstrated to prevent such bone loss and either maintain or further increase bone mass [19, 22]. Based on these observations, the current study was undertaken to examine the effects of bisphosphonate treatment (1 year of alendronate) on bone mass and bone quality in patients with chronic SCI after the discontinuation of anabolic therapy (1 or 2 years with teriparatide). We expected to observe either maintenance or increase in bone measures, over the course of the study.

\section{Methods}

\section{Study design}

This study was a single-site, non-randomized, open-label clinical trial conducted from April 2014 to August 2016 to determine if alendronate would be effective in maintaining the BMD increase observed after at least 12 months of teriparatide therapy. Invitation to enroll in this 12-month study was only offered to the group of participants with chronic SCI, who had recently completed a randomized controlled trial of teriparatide and/or vibration therapy (NCT01225055) and its open-label teriparatide extension (NCT02025179). These two teriparatide treatment clinical trials have been previously reported by our group [18] and hereafter, will be referred to as the parent protocol or parent study.

All visits occurred at Northwestern University Feinberg School of Medicine (NU) and the Rehabilitation Institute of Chicago (RIC), now known as the Shirley Ryan AbilityLab. Bone quality assessments mirrored those of the lead site as described in the parent protocol [18]. This study was registered with ClinicalTrials.gov (NCT02195895) and was approved by the Northwestern University Institutional Review Board (IRB) and DOD's Human Research Protection Office (HRPO). Informed consent was obtained prior to the start of any study procedures.

\section{Study participants}

To be eligible for the parent protocol [18], participants (male or female) were required to be at least 21 years old, and non-ambulatory following a history of SCI, classified based on chart review or assessment by the referring physician. They were also required to have (1) low bone mass at the total hip or femoral neck based on dual-energy absorptiometry (DXA), with a $Z$-score $\leq-1.5, T$-score $\leq$ -2.5 , or $T$-score $<-2.0$ with a history of a fragility fracture, (2) a SCI at least 1 year before the start of the study, (3) an ASIA impairment score of A, B, C, or D (non-ambulatory), (4) normal renal function, $\mathrm{TSH}$, and calcium levels, and (5) $25-\mathrm{OH}$ Vitamin D levels $\geq 20 \mathrm{ng} / \mathrm{ml}$ at baseline. Individuals were excluded if they (1) had known allergies to teriparatide or a history of teriparatide use (last 24 months), (2) had a history of bone metastasis, radiation therapy, or Paget's disease of bone, (3) used anticonvulsants at a frequency determined to interfere with bone metabolism, (4) had elevated liver function (more than two times normal limit), and (5) were pregnant, lactating, or planning on becoming pregnant.

Out of the 24 individuals who completed the parent protocol and received 1 or 2 years of teriparatide therapy, 17 agreed to participate in the current study. Of the seven who did not continue, only one cited a medically relevant reason, as they were not able to sit up for $30 \mathrm{~min}$ to safely take the drug.

\section{Study treatment and procedures}

Each participant from the parent protocol was further screened to ensure safety prior to enrolling in this openlabel study. Major exclusion criteria included: (1) renal insufficiency, (2) not able to sit upright for at least $30 \mathrm{~min}$ after taking study medication, (3) poor dental hygiene or plans for dental surgery in the next year, and (4) esophageal abnormalities.

After passing the screening procedures, participants were carefully instructed in the proper way to take the study drug (first thing in the morning, sitting upright, on an empty stomach with a full glass of water, etc.). Participants received study drug (alendronate $70 \mathrm{mg}$ ) for weekly use and supplemental vitamin D (cholecalciferol $1000 \mathrm{IU})$ and calcium (calcium carbonate $1000 \mathrm{mg}$ ) for daily use. Study drug and supplements were provided in sufficient quantities to last until the next clinic visit. Follow-up study visits 
occurred every 3 months after the initiation of study treatment over the course of 12 months. Adverse events and compliance were assessed at each visit.

Bone quality assessments were collected at baseline, and again at 6 and 12 month after starting alendronate therapy, and included DXA scans of the lumbar spine and bilateral hips, computed tomography (CT) of the non-dominant knee, and serum bone markers (collagen type 1 cross-linked C-telopeptide [CTX], type 1 procollagen amino-terminal propeptide [P1NP], and bone-specific alkaline phosphatase [BSAP]). Since enrollment into this study began shortly after the completion of the parent protocol and the bone quality assessments were identical in all studies, the final (month 24) visit of the parent protocol was used as the baseline visit for this study. If more than 3 months elapsed between the studies, bone serum markers, DXA and CT imaging were repeated prior to the start of study treatment.

\section{DXA acquisition and analysis}

All scans were performed with a Hologic QDR 4500A (Hologic, Waltham, MA, USA) as previously described [23]. Standard acquisition and analysis protocols were used to quantify areal BMD (aBMD) of the lumbar spine, total hip and femoral neck, from frontal plane images. Quality control of the DXA machine included measurements of the spine phantom daily, body phantom three times/week, and air scan and tissue bar scans weekly. The non-dominant hip data (total hip and femoral neck) were chosen for analysis. For participants with significant heterotopic ossification (HO) or other artifacts in the region of interest, the dominant hip data were used.

\section{CT acquisition and analysis}

CT image acquisition and analysis were performed according to a previously published protocol [18], repeated here briefly. Scans of the knee were acquired using a Sensation 64 Cardiac Scanner (Siemens Medical Systems, Forchheim, Germany), with scan settings of $120 \mathrm{kVp}$ and $280 \mathrm{~mA}$. Images were acquired with an in-plane resolution of $0.352 \times 0.352 \mathrm{~mm}$ with a $1 \mathrm{~mm}$ slice spacing. A $30 \mathrm{~cm}$ long scan was used to capture $\sim 15 \mathrm{~cm}$ of the proximal tibia and distal femur, respectively. A hydroxyapatite calibration phantom was placed in the field-of-view, which allowed us to identify a linear regression relationship between mineral density $\left(\mathrm{g} / \mathrm{cm}^{3}\right)$ and CT absorption (HU; Hounsfield units).

Regions of interest from each scan were identified using Mimics (Materialise, Leuven, Belgium) software. A threshold of $0.15 \mathrm{~g} / \mathrm{cm}^{3}$ was used to identify the periosteal surface of the bone, and some manual clean-up was done to isolate each bone and fill in small gaps at the epiphysis. Images from the baseline assessment, prior to any treatment, were manually aligned along the longitudinal axes of the tibia and femur. Scans from each follow-up visit were registered to their corresponding aligned baseline images using a least-squares optimization algorithm available in the Mimics software.

Aligned scans of each bone were separated into different regions for CT analysis. First, segment lengths (SL) were estimated from published proportionality constants [24] and self-reported stature. Segment lengths were then used to separate each bone into epiphyseal (0-10\% SL), metaphyseal (10-20\% SL), and diaphyseal (20-30\% SL) regions. Each region was further separated into integral, trabecular, and cortical compartments. The integral compartment contained all voxels within the periosteal surface. The trabecular compartment was identified by applying a 10-pixel $(3.52 \mathrm{~mm})$ in-plane erosion to the integral compartment. Finally, cortical bone was identified by Boolean subtraction of the trabecular region from the integral region, followed by a thresholding of $0.35 \mathrm{~g} / \mathrm{cm}^{3}$ to remove any remaining trabecular bone. We reported volumetric bone mineral density (vBMD; $\mathrm{g} / \mathrm{cm}^{3}$ ) for trabecular bone and bone mineral content $(\mathrm{BMC} ; \mathrm{g})$ and bone volume $\left(\mathrm{BV} ; \mathrm{cm}^{3}\right)$ for cortical bone. The trabecular compartment was omitted from analysis of the diaphyseal region. Finally, we computed a compressive strength index $\left(\mathrm{CSI} ; \mathrm{g}^{2} / \mathrm{cm}^{4}\right)$ and torsional strength index (TSI; $\mathrm{cm}^{3}$ ) using previously reported equations $[25,26]$.

\section{Finite element analysis}

We also generated CT-based, subject-specific finite element (FE) models of each bone, in order to assess torsional stiffness and strength. Briefly, images were resampled to isotropic $1.5 \mathrm{~mm}$ resolution voxels and a hexahedral mesh of the same size was generated by voxel conversion. Material properties were assigned based on average CT intensity at each element location using equation 1 :

$$
\begin{aligned}
& E_{3}=6570 \cdot \rho_{\text {app }}^{1.37} \\
& \rho_{\text {app }}=\frac{\rho_{\mathrm{HU}}}{0.626}
\end{aligned}
$$

where $E_{3}$ is the Young's modulus in the proximal-distal direction (MPa), $\rho_{\text {app }}$ is the apparent density $\left(\mathrm{g} / \mathrm{cm}^{3}\right)$ and $\rho_{\text {HU }}$ is the CT-derived density $\left(\mathrm{g} / \mathrm{cm}^{3}\right)$. The other elastic constants were computed using previously published ratios of anisotropy: $E_{1}=0.574 \cdot E_{3}, \quad E_{2}=0.577 \cdot E_{3}$, $G_{12}=0.195 \cdot E_{3}, \quad G_{23}=0.265 \cdot E_{3}, \quad G_{31}=0.216 \cdot E_{3}, \quad \nu_{12}=$ $0.427, \nu_{23}=0.234$, and $\nu_{31}=0.405$ [27]. Here subscripts 1,2 , and 3 denote the medial-lateral, anterior-posterior, and proximal-distal directions, respectively. Bone material failure was simulated using a bilinear elastic-plastic model, where yield was defined with a quadratic Hill criterion for 
orthotropic materials [28]. Surface nodes on $2 \mathrm{~cm}$ of the diaphyseal end were rigidly fixed, while torsional displacement was applied to surface nodes on $2 \mathrm{~cm}$ of the epiphyseal end. Torsional stiffness $\left(K_{\mathrm{t}}\right)$ was quantified from the linear region of the simulated torque-rotation response. Torsional strength $\left(T_{\mathrm{ult}}\right)$ was computed as the applied torque required to cause $10 \%$ of the surface to exceed a maximum principle strain of $1.41 \%$ [29]. This modeling procedure was thoroughly validated using cadaveric tibiae [29], and has been used to study individuals with SCI [18, 30, 31].

\section{Statistical analysis}

Statistical analyses were performed using SPSS software (version 24, IBM, NY, USA). The primary outcome measure was percent change in DXA BMD at the total hip at 12 months. Secondary outcome measures included changes in BMD at other skeletal sites, changes in CT-defined skeletal outcome measures and serum biomarker changes. Paired Student's $t$-tests were performed at a significance level of 0.05 , comparing the value of each measure at follow-up against the baseline measured prior to treatment with alendronate. To confirm validity of this test, we assessed the normality of paired differences using the Shapiro-Wilks tests evaluated at a significance level of 0.05 .

\section{Results}

\section{Participant flow}

Seventeen participants who completed the parent protocol agreed to participate and qualified to enroll in this openlabel extension study. Thirteen participants completed the study per protocol. Four participants had to discontinue medication for varying lengths of time during the 12 months. One was on bed rest for a pressure sore, and unable to sit up for $30 \mathrm{~min}$ to safely take the drug. Two were taken off study drug due to a potential need for surgical tooth extraction, and one required fenestration of a spine syrinx (around week 24) and experienced medical complications. However, all returned for a final visit. Sixteen participants used the therapy for $>76 \%$ of the 12-month duration of this study, while one used study drug for only $21 \%$ of the duration of this study. This individual was assessed as a possible outlier, but excluding their data did not have a meaningful impact on the significance of the results. Thus, all participants were included in this report.

\section{Participant demographic and clinical descriptors}

As described in Table 1, the study population was predominantly male (76\%). About half of the participants were
Table 1 Patient demographics and clinical descriptors

\begin{tabular}{|c|c|}
\hline \multicolumn{2}{|l|}{ Open-label cohort, $n$} \\
\hline Participants & 17 \\
\hline \multicolumn{2}{|l|}{ Age, mean (SD) } \\
\hline Years & $44(13)$ \\
\hline \multicolumn{2}{|l|}{ Gender, $n(\%)$} \\
\hline Male & $13(76)$ \\
\hline Female & $4(24)$ \\
\hline \multicolumn{2}{|l|}{ Ethnicity, $n(\%)$} \\
\hline Non-Hispanic & $14(82)$ \\
\hline Hispanic or Latino & $3(18)$ \\
\hline \multicolumn{2}{|l|}{ Race, $n(\%)$} \\
\hline Caucasian & $9(53)$ \\
\hline African-American & $7(41)$ \\
\hline Asian & $1(6)$ \\
\hline \multicolumn{2}{|l|}{ Time since injury, mean (SD) } \\
\hline Years & $15(9)$ \\
\hline \multicolumn{2}{|l|}{ Spine region, $n(\%)$} \\
\hline Cervical & $4(24)$ \\
\hline Thoracic & $11(65)$ \\
\hline Lumbar & $2(12)$ \\
\hline \multicolumn{2}{|l|}{ AIS Classification, $n(\%)$} \\
\hline A & $12(71)$ \\
\hline B & $1(6)$ \\
\hline $\mathrm{C}$ & $4(24)$ \\
\hline \multicolumn{2}{|l|}{ Injury severity, $n(\%)$} \\
\hline Motor complete tetraplegia (>T1) & $3(17)$ \\
\hline Motor complete paraplegia $(<\mathrm{C} 8)$ & $10(58)$ \\
\hline Motor incomplete tetraplegia (>T1) & $1(6)$ \\
\hline Motor incomplete paraplegia $(<\mathrm{C} 8)$ & $3(18)$ \\
\hline \multicolumn{2}{|l|}{ History of smoking, $n(\%)$} \\
\hline Yes & $4(23)$ \\
\hline No & $13(75)$ \\
\hline \multicolumn{2}{|l|}{ History of fracture after SCI, $n(\%)$} \\
\hline Prior to participation & $5(29)$ \\
\hline During study & $1(5)$ \\
\hline None & $11(65)$ \\
\hline \multicolumn{2}{|l|}{ Hip aBMD at baseline, mean (SD) } \\
\hline Z-Score & $-2.5(0.8$ \\
\hline
\end{tabular}

Caucasian (53\%) and just under half were AfricanAmerican (41\%). Thoracic level lesions were most common $(65 \%)$, and approximately three-quarters of the patients were classified as American Spinal Injury Association Impairment Scale (AIS) A or B and had a motor complete injury. Four participants had a history of smoking. Finally, five participants had experienced at least one incident fracture after SCI and prior to the start of this study. One participant experienced a fracture while in the study; this individual was also assessed as a possible outlier. Excluding 
their data had little impact on the significance of our results, and so all data were kept in the final analysis.

\section{DXA outcomes}

DXA measurements were analyzed for all 17 participants, with the exception of one individual's total hip being omitted due to HO. As shown in Table 2 and Fig. 1, 6 months of treatment with alendronate was not associated with a significant change to aBMD at the hip, femoral neck, or spine, with mean changes of $0.27 \%$ (95\% CI, -1.7 to $2.2), 1.8 \%$ (95\% CI, -1.0 to 4.6 ), and -1.6 (95\% CI, -3.3 to 0.05 ), respectively. After 12 months of treatment, changes at the hip and femoral neck remained non-significant, with changes of $1.3 \%$ (95\% CI, -1.1 to 3.8 ) and $0.54 \%$ (95\% CI, -2.0 to 3.1), respectively. However, a significant increase in mean spine aBMD $(2.5 \%$; $95 \% \mathrm{CI}, 0.17$ to 4.9$)$ was demonstrated after 12 months.

\section{CT and FE outcomes}

As shown in Table 2 and Fig. 1, we observed statistically significant improvements in a number of CT measures at the distal femur. After 6 months, cortical BMC increased by $14 \%$ (95\% CI, 3.8 to 24) and cortical BV increased by $13 \%$ (95\% CI, 4.0 to 23 ) at the epiphysis. Further improvements were seen after 12 months. At this time-point, cortical BMC increased by $15 \%$ (95\% CI, 5.5 to 24 ), $7.7 \%$ (95\% CI, 1.3 to 14), and $3.0 \%$ (95\% CI, 1.2 to 4.8 ) at the epiphysis, metaphysis, and diaphysis, respectively. Similarly, there were also improvements to cortical $\mathrm{BV}$ at the epiphysis (15\%; 95\% CI, 5.8 to 23$)$ and TSI of the metaphysis (4.6\%; $95 \% \mathrm{CI}, 1.1$ to 8.2 ). However, a small decrease in diaphyseal cortical BV was also observed at 6 months $(-4.3 \%$; 95\% CI, -5.6 to -3.0$)$ and 12 months $(-3.2 \%$; $95 \% \mathrm{CI}$, -4.3 to -2.1$)$.

Improvements were somewhat diminished at the proximal tibia (Table 2). At this skeletal site we only observed increases in cortical BMC (10\%; 95\% CI, 3.0 to 17) and cortical BV (7\%; 95\% CI, 0.63 to 13$)$ at the epiphysis after 12 months. However, cortical BV decreased in the metaphysis and diaphysis, with changes of $-4.0 \%(95 \% \mathrm{CI}$, -6.4 to -1.7$)$ and $-3.7 \%$ (95\% CI, -5.2 to -2.2$)$, respectively. Similar changes in these measures were observed at the 6-month follow-up (Table 2), but we also observed that metaphyseal trabecular BMD decreased by $-127 \%$ (95\% CI, -219 to -34 ) after 12 months. However, even at baseline, trabecular BMD had a mean (SD) of $-0.002(0.043) \mathrm{g} / \mathrm{cm}^{3}$. The negative score here indicates that many individuals had little to no trabecular bone in this region even at the start of the study, and a reduction $>100 \%$ suggests a region that was entirely composed of marrow fat with little remaining hydroxyapatite after 12 months. No other changes were statically significant. We also did not observe statistically significant changes in FE-derived torsional stiffness $(p>0.21)$ or strength $(p>0.52)$ at the tibia at either the 6 or 12-month follow-up visits (Table 2 and Fig. 2).

\section{Biomarker outcomes}

Biomarker data were analyzed for all 17 participants. CTX, P1NP, and BSAP were all significantly lower after 6 months, with changes of $-61 \%$ (95\% CI, -92 to -29$)$, $-59 \%$ ( $95 \% \mathrm{CI},-88$ to -29$)$, and $-34 \%$ ( $95 \% \mathrm{CI},-54$ to $-14)$, respectively. After 12 months, CTX remained $62 \%$ lower than baseline (95\% CI, -100 to -24$)$ and BSAP remained 52\% lower than baseline (95\% CI, -94 to -9.3 ), but changes to BSAP were no longer significant $(p=0.36)$.

\section{Safety outcomes}

Eighty-two percent of participants reported at least one adverse event during the course of the study. Eight of the adverse events reported were serious (bladder stone, inferior vena cava shift, two cases of sepsis secondary to an urinary tract infection (UTI), pressure sore, infection at the site of a pressure sore, spinal cord cysts, and two UTIs); however, none was felt to be related to study interventions.

\section{Discussion}

The results of this study indicate that one year of treatment with alendronate following discontinuation of treatment with teriparatide in people with SCI resulted in maintenance of bone mass at most skeletal sites. Alendronate therapy prevented the decrease in DXA-determined aBMD that has been reported to occur in able-bodied individuals after cessation of an anabolic intervention such as teriparatide therapy [19-21, 32, 33]. Not only did 12 months of alendronate therapy prevent bone loss, but a statistically significant increase in aBMD was observed at the spine and numerical increases in aBMD were demonstrated at the hip. However, these are less common sites of fracture in individuals with SCI, therefore these changes may not be clinically meaningful. Results were more variable at the distal femur and proximal tibia, the most common sites for fracture in this population. We observed increases in some skeletal parameters and bone strength indices at the distal femur, but with mixed results at the tibia.

Until recently, teriparatide has been the only anabolic agent approved to treat osteoporosis in post-menopausal women and older, osteoporotic men [32, 33]. An additional anabolic agent, abaloparatide, a parathyroid-related protein 


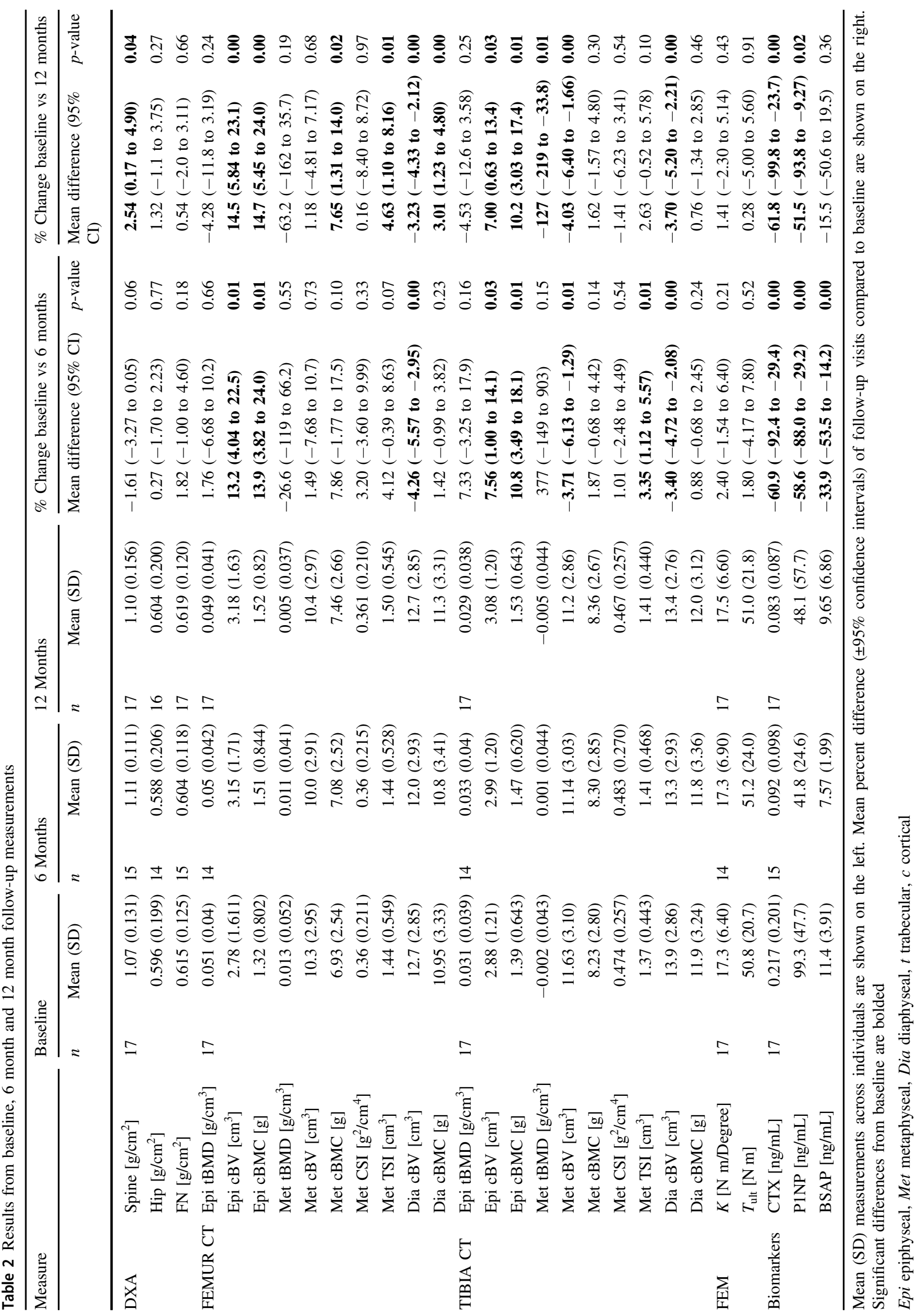



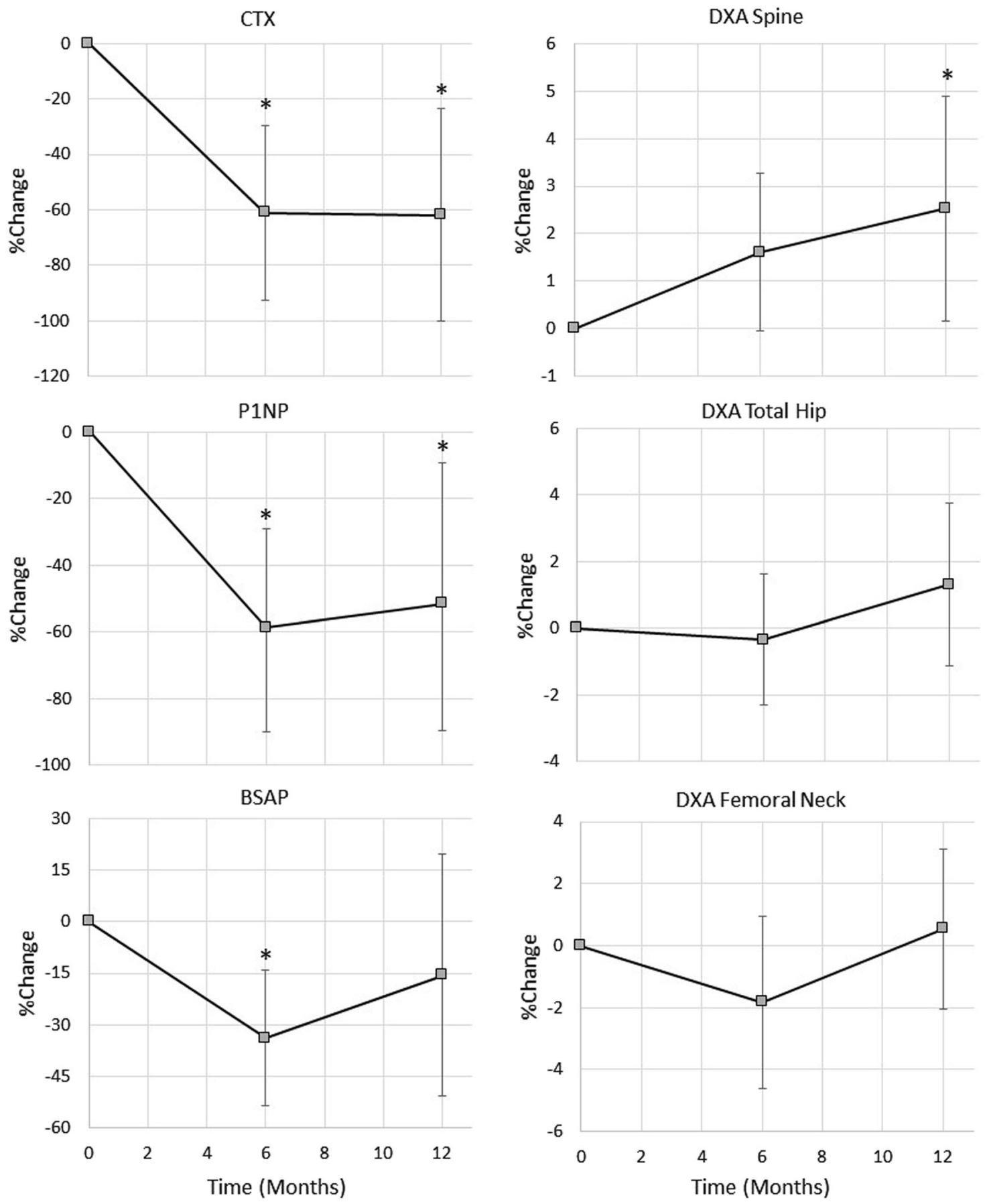

Fig. 1 Mean values ( $\pm 95 \%$ confidence intervals) for serum biomarkers of bone metabolism (left) and select DXA measurements (right) relative to baseline

analog, has also been approved for the treatment of post-menopausal osteoporosis and shown to reduce the risk of vertebral and non-vertebral fractures over 18 months of treatment [34]. Because of the marked skeletal bone loss in many people with long-standing SCI, these anabolic agents would seem to be preferred approaches to rebuilding bone mass and bone strength, thereby reducing fracture risk in this population.
Based on the findings of teriparatide treatment in ablebodied individuals, we initiated and recently completed a randomized controlled trial of teriparatide in 60 people with chronic SCI and low aBMD; this study demonstrated small increases in aBMD at the spine and more limited increases at the hip after 1 and 2 years of treatment [18]. As teriparatide treatment is limited to 2 years, and the increases in bone mass and bone strength were modest, the opportunity 


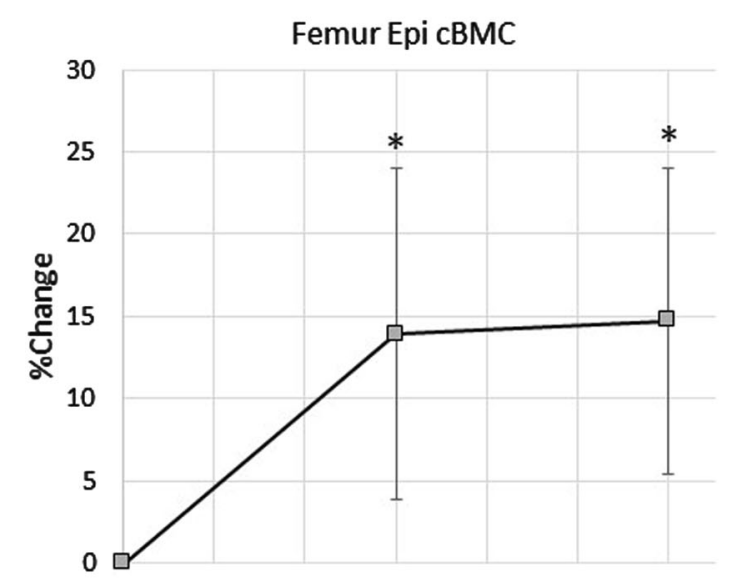

Tibia Epi cBMC

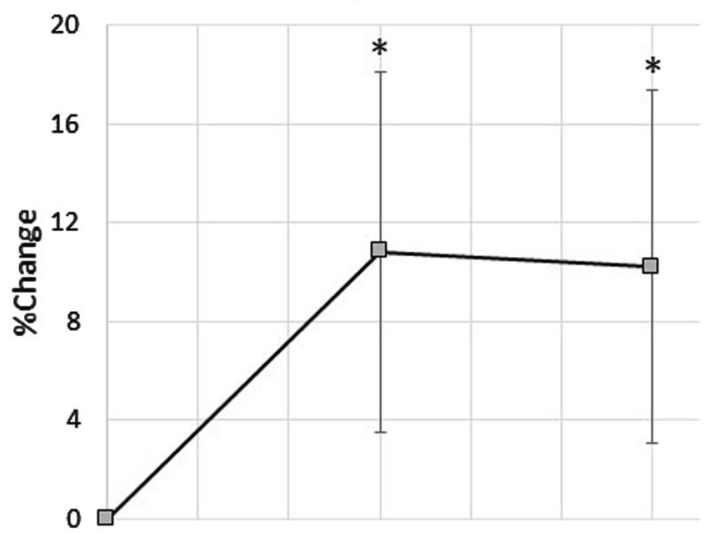

Tibia Finite Element $\mathrm{K}$

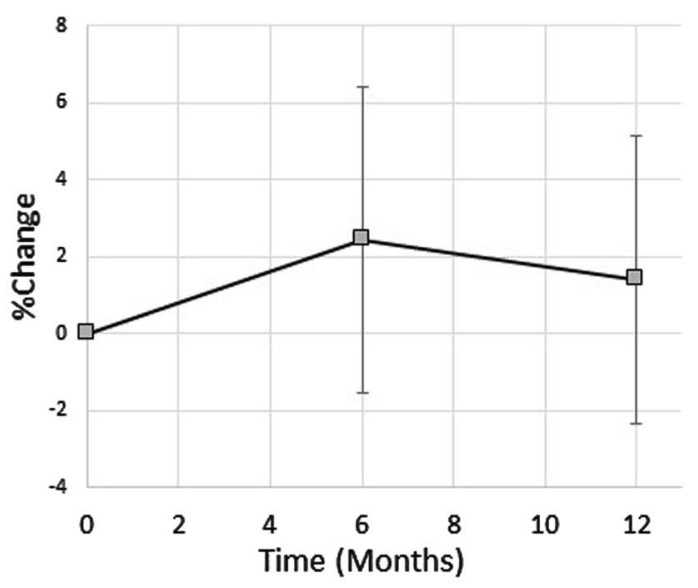

Fig. 2 Mean values ( $\pm 95 \%$ confidence intervals) for select CT measurements at the distal femur (top), select CT measurements at the

arose to determine if a further year of an oral bisphosphonate, alendronate, would result in maintaining the skeletal benefits of anabolic therapy and perhaps also be associated with further gains in bone mass and/or bone strength. Previous studies with teriparatide in postmenopausal women have demonstrated that discontinuation of teriparatide treatment was followed by loss of
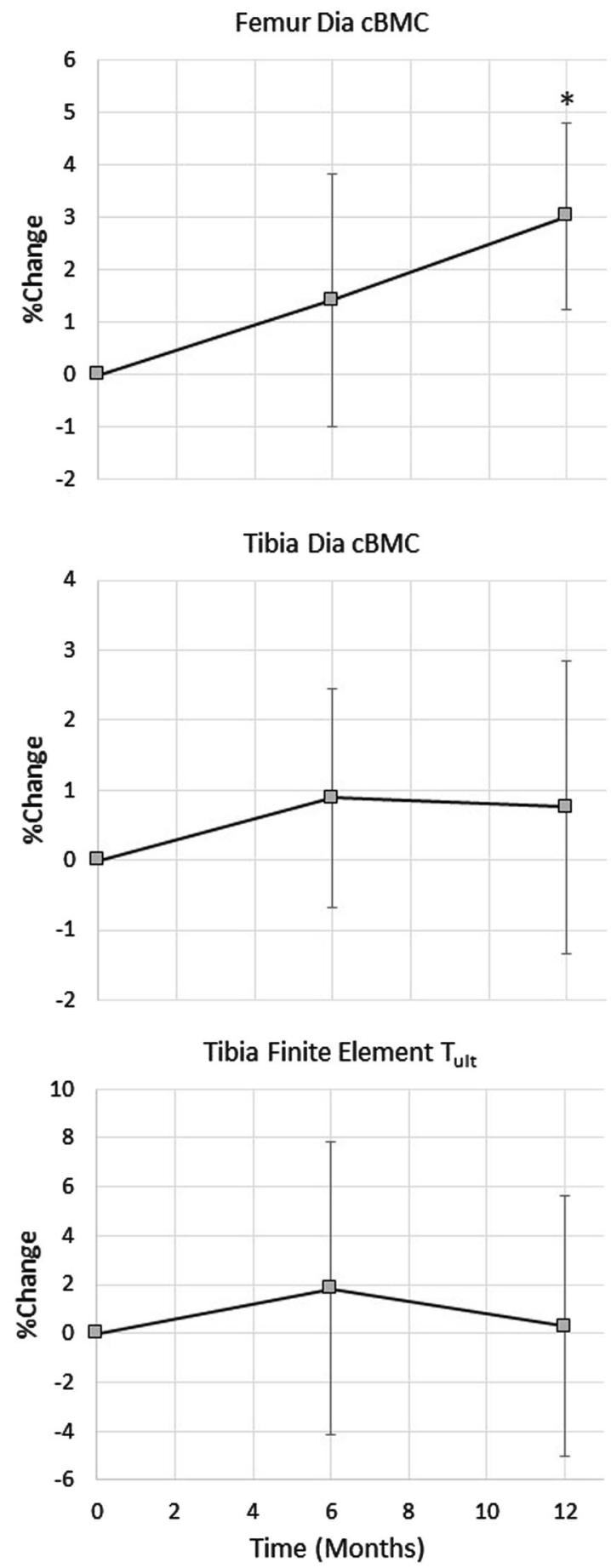

proximal tibia (middle), and finite element predictions of stiffness $(K)$ and strength $\left(T_{\text {ult }}\right)$ at the proximal tibia (bottom)

aBMD, and that this loss could be prevented by the use of antiresorptive therapy [19, 35]. Results of this study support this notion, as we observed that treatment with alendronate after teriparatide resulted in small gains, or no significant change, at the spine, hip, and many sites of the knee. Reductions were observed at the tibia, a common fracture site in individuals with SCI, but FE modeling suggested no 
significant change in bone stiffness or strength at this location. This suggests that these small reductions did not have important effects on the mechanical competence of the bones.

Teriparatide administration results in an increased bone anabolic activity, reflected in an increase in serum bone markers of bone formation such as P1NP and BSAP. However, there is also a marked increase in serum markers of bone catabolic activity (e.g., CTX) with use of teriparatide [36]. After discontinuation of teriparatide, bone formation decreases significantly while bone resorption remains elevated, resulting in subsequent bone loss [19]. The use of bisphosphonates, which work by inhibiting bone resorption, would be expected to prevent this bone loss. This finding has been reported in post-menopausal women after teriparatide treatment $[19,35]$ and in the current study. Elevated biomarkers of bone turnover, present during teriparatide treatment, were markedly reduced with alendronate therapy in this study. The prevention of bone loss after cessation of teriparatide has also been reported with the use of denosumab [37], an antibody to RANK ligand, which works to inhibit osteoclastogenesis and subsequent bone resorption.

The knee represents the skeletal site most prone to fracture after SCI, and therefore the observed additional improvements in CT-based skeletal parameters at the distal femur following alendronate therapy was encouraging. Although some CT-based measurements at the proximal tibia illustrated significant declines following alendronate therapy (e.g., trabecular BMD and cortical $\mathrm{BV}$ at the metaphysis), FE-derived measurements of torsional stiffness and strength were unchanged from the final visit of the parent study, which demonstrated increases in FE-derived stiffness and of $5.0 \%$ and $7.4 \%$ [18], respectively, suggesting that improvements to skeletal strength were maintained. It is important to note that the finding of a small but beneficial effect of alendronate after cessation of teriparatide therapy for people with SCI and bone loss does not mean that a similar benefit would be seen in a clinical setting without previous anabolic treatment. In chronic SCI, bone formation is depressed, with lower than normal levels of serum markers of anabolic activity $[9,38]$. Therefore, additional inhibition of bone formation with an antiresorptive agent, even in the presence of inhibition of bone resorption, would not be expected to provide much, if any, benefit. Indeed, this has been the finding in the majority of studies done to date [39-41]. On the other hand, Zehnder et al. [42] suggested that treatment with alendronate could prevent bone loss without previous anabolic treatment, though it has been suggested that the discrepancy between studies could be due to differences in patient age and lower injury severity [41].
This study has a few noteworthy limitations. This was the second extension offered to individuals in the parent study, and many chose not to continue participating at the end of each stage. As a result, the sample size is quite modest. Furthermore, there were not enough individuals to have a control arm, though this limitation is somewhat mitigated by the fact that it has already been established that cessation of anabolic treatment is associated with a rapid loss of previously gained bone [19-22]. As a result of this small sample size, however, we are unable to draw meaningful conclusions about potential confounding variables such as sex or injury severity.

To summarize, in the setting of completion of a course of bone anabolic therapy, an antiresorptive agent such as alendronate, may provide additional skeletal benefit to people with SCI. With new and more potent anabolic agents available and being actively investigated [34, 43, 44], it may yet be possible to increase and maintain improved bone mass and bone strength in people with long-standing SCI. Though more investigation is required, such treatments could result in a decrease in fracture risk and fracture prevalence in individuals with SCI.

\section{Data archiving}

The datasets generated and/or analyzed in this study are available from the corresponding author on reasonable request.

Acknowledgements We would like to thank Stacey M. Lobos for assisting with CT image analysis.

Funding This research was supported by Department of Defense U.S. Army Medical Research and Materiel Command [Grant \#SC090010] and the National Institutes of Health's National Center for Advancing Translational Sciences [Grant Numbers UL1TR001422 and UL1TR000150]. The content is solely the responsibility of the authors and does not necessarily represent the official views of these funding agencies.

Author contributions IH: Performed CT image analysis, FE modeling, and statistical analysis of the data. He also helped draft the portions of the initial report. NS: Responsible for data collection, data analysis, and drafting and revising the report. AS: Responsible for data collection, data analysis, and revising the report. FL: Responsible for data collection, data analysis, and revising the report. WBE: Helped with study design. Supervised CT image analysis, FE modeling, and statistical analysis. He was also responsible for interpretation of the data. TS: Designed the study and supervised data collection and statistical analysis tasks. He was also responsible for interpretation of the data. All authors helped to revise the initial report and approved the final submission.

\section{Compliance with ethical standards}

Conflict of interest The authors declare that they have no conflict of interest. 
Statement of ethics This study was registered with ClinicalTrials.gov (NCT02195895) and was approved by the Northwestern University Institutional Review Board (IRB) and DOD's Human Research Protection Office (HRPO). Informed consent was obtained prior to the start of any study procedures.

Publisher's note: Springer Nature remains neutral with regard to jurisdictional claims in published maps and institutional affiliations.

\section{References}

1. Biering-Sorensen F, Bohr HH, Schaadt OP. Longitudinal study of bone mineral content in the lumbar spine, the forearm and the lower extremities after spinal cord injury. Eur J Clin Invest. 1990;20:330-5.

2. Coupaud S, McLean AN, Purcell M, Fraser MH, Allan DB. Decreases in bone mineral density at cortical and trabecular sites in the tibia and femur during the first year of spinal cord injury. Bone. 2015;74:69-75.

3. Frey-Rindova P, de Bruin ED, Stussi E, Dambacher MA, Dietz V. Bone mineral density in upper and lower extremities during 12 months after spinal cord injury measured by peripheral quantitative computed tomography. Spinal Cord. 2000;38:26-32.

4. Frotzler A, Berger M, Knecht H, Eser P. Bone steady-state is established at reduced bone strength after spinal cord injury: a longitudinal study using peripheral quantitative computed tomography (pQCT). Bone. 2008;43:549-55.

5. Eser P, Schiessl H, Willnecker J. Bone loss and steady state after spinal cord injury: a cross-sectional study using pQCT. J Musculoskelet Neuron Interact. 2004;4:197-8.

6. Battaglino RA, Lazzari AA, Garshick E, Morse LR. Spinal cord injury-induced osteoporosis: pathogenesis and emerging therapies. Curr Osteoporos Rep. 2012;10:278-85.

7. Dionyssiotis Y, Trovas G, Galanos A, Raptou P, Papaioannou N, Papagelopoulos $\mathrm{P}$, et al. Bone loss and mechanical properties of tibia in spinal cord injured men. J Musculoskelet Neuron Interact. 2007;7:62-8.

8. Lazo MG, Shirazi P, Sam M, Giobbie-Hurder A, Blacconiere MJ, Muppidi M. Osteoporosis and risk of fracture in men with spinal cord injury. Spinal Cord. 2001;39:208-14.

9. Lipscomb SD, Zehnder SM. Immersion in the virtual environment: the effect of a musical score on the video gaming experience. J Physiol Anthr Appl Hum Sci. 2004;23:337-43.

10. Gifre L, Vidal J, Carrasco J, Portell E, Puig J, Monegal A, et al. Incidence of skeletal fractures after traumatic spinal cord injury: a 10-year follow-up study. Clin Rehabil. 2014;28:361-9.

11. Nottage WM. A review of long-bone fractures in patients with spinal cord injuries. Clin Orthop Relat Res. 1981;155:65-70.

12. Morse LR, Battaglino RA, Stolzmann KL, Hallett LD, Waddimba A, Gagnon D, et al. Osteoporotic fractures and hospitalization risk in chronic spinal cord injury. Osteoporos Int. 2009;20:385-92.

13. Carbone LD, Chin AS, Burns SP, Svircev JN, Hoenig H, Heggeness $\mathrm{M}$, et al. Morbidity following lower extremity fractures in men with spinal cord injury. Osteoporos Int. 2013;24:2261-7.

14. Frotzler A, Cheikh-Sarraf B, Pourtehrani M, Krebs J, Lippuner K. Long-bone fractures in persons with spinal cord injury. Spinal Cord. 2015;53:701-4.

15. Bethel M, Weaver FM, Bailey L, Miskevics S, Svircev JN, Burns SP, et al. Risk factors for osteoporotic fractures in persons with spinal cord injuries and disorders. Osteoporos Int. 2016;27:3011-21.

16. Neer RM, Arnaud CD, Zanchetta JR, Prince R, Gaich GA, Reginster JY, et al. Effect of parathyroid hormone (1-34) on fractures and bone mineral density in postmenopausal women with osteoporosis. N Engl J Med. 2001;344:1434-41.

17. Kleerekoper M, Greenspan SL, Lewiecki EM, Miller PD, Kendler DL, Maricic M, et al. Assessing the effects of teriparatide treatment on bone mineral density, bone microarchitecture, and bone strength. J Bone Jt Surg Am. 2014;96:e90.

18. Edwards WB, Simonian N, Haider IT, Anschel AS, Chen D, Gordon KE, et al. Effects of teriparatide and vibration on bone mass and bone strength in people with bone loss and spinal cord injury: a randomized, controlled trial. J Bone Min Res. 2018;33:1729-40.

19. Black DM, Bilezikian JP, Ensrud KE, Greenspan SL, Palermo L, Hue $\mathrm{T}$, et al. One year of alendronate after one year of parathyroid hormone (1-84) for osteoporosis. N Engl J Med. 2005;353:555-65.

20. Leder BZ, Neer RM, Wyland JJ, Lee HW, Burnett-Bowie SM, Finkelstein JS. Effects of teriparatide treatment and discontinuation in postmenopausal women and eugonadal men with osteoporosis. J Clin Endocrinol Metab. 2009;94:2915-21.

21. Cohen A, Kamanda-Kosseh M, Recker RR, Lappe JM, Dempster $\mathrm{DW}$, Zhou $\mathrm{H}$, et al. Bone density after teriparatide discontinuation in premenopausal idiopathic osteoporosis. J Clin Endocrinol Metab. 2015;100:4208-14.

22. Prince R, Sipos A, Hossain A, Syversen U, Ish-Shalom S, Marcinowska E, et al. Sustained nonvertebral fragility fracture risk reduction after discontinuation of teriparatide treatment. J Bone Min Res. 2005;20:1507-13.

23. Gordon KE, Wald MJ, Schnitzer TJ. Effect of parathyroid hormone combined with gait training on bone density and bone architecture in people with chronic spinal cord injury. PM R. 2013;5:663-71.

24. Winter DA. Biomechanics and motor control of human movement. 4th ed. Hoboken, N.J.: Wiley; 2009. p. 370. xiv

25. Lang T, LeBlanc A, Evans H, Lu Y, Genant H, Yu A. Cortical and trabecular bone mineral loss from the spine and hip in longduration spaceflight. J Bone Min Res. 2004;19:1006-12.

26. Edwards WB, Schnitzer TJ, Troy KL. Bone mineral and stiffness loss at the distal femur and proximal tibia in acute spinal cord injury. Osteoporos Int. 2014;25:1005-15.

27. Rho J-Y. An ultrasonic method for measuring the elastic properties of human tibial cortical and cancellous bone. Ultrasonics. 1996;34:777-83.

28. Hill R. A theory of the yielding and plastic flow of anisotropic metals. Proc R Soc Lond Ser A, Math Phys Sci. 1948;193:281-97.

29. Edwards WB, Schnitzer TJ, Troy KL. Torsional stiffness and strength of the proximal tibia are better predicted by finite element models than DXA or QCT. J Biomech. 2013;46:1655-62.

30. Edwards WB, Simonian N, Troy KL, Schnitzer TJ. Reduction in torsional stiffness and strength at the proximal tibia as a function of time since spinal cord injury. J Bone Min Res. 2015;30:1422-30.

31. Edwards WB, Schnitzer TJ, Troy KL. The mechanical consequence of actual bone loss and simulated bone recovery in acute spinal cord injury. Bone . 2014;60:141-7.

32. Cosman F. Parathyroid hormone treatment for osteoporosis. Curr Opin Endocrinol Diabetes Obes. 2008;15:495-501.

33. Whitfield JF, Morley P, Willick GE. Parathyroid hormone, its fragments and their analogs for the treatment of osteoporosis. Treat Endocrinol. 2002;1:175-90.

34. Miller PD, Hattersley G, Riis BJ, Williams GC, Lau E, Russo LA, et al. Effect of abaloparatide vs placebo on new vertebral fractures in postmenopausal women with osteoporosis: a randomized clinical trial. Jama. 2016;316:722-33.

35. Leder BZ, Tsai JN, Jiang LA, Lee H. Importance of prompt antiresorptive therapy in postmenopausal women discontinuing teriparatide or denosumab: the Denosumab and Teriparatide Follow-up study (DATA-Follow-up). Bone. 2017;98:54-8.

36. Black DM, Greenspan SL, Ensrud KE, Palermo L, McGowan JA, Lang TF, et al. The effects of parathyroid hormone and 
alendronate alone or in combination in postmenopausal osteoporosis. N Engl J Med. 2003;349:1207-15.

37. Leder BZ, Tsai JN, Uihlein AV, Wallace PM, Lee H, Neer RM, et al. Denosumab and teriparatide transitions in postmenopausal osteoporosis (the DATA-Switch study): extension of a randomised controlled trial. Lancet. 2015;386:1147-55.

38. Reiter AL, Volk A, Vollmar J, Fromm B, Gerner HJ. Changes of basic bone turnover parameters in short-term and long-term patients with spinal cord injury. Eur Spine J. 2007;16:771-6.

39. Vukusich A, Alvear F, Villanueva P, Gonzalez C, Francisco O, Alvarado N, et al. Epidemiology of severe acute renal failure in Metropolitan Santiago. Rev Med Chil. 2004;132:1355-61.

40. Moran de Brito CM, Battistella LR, Saito ET, Sakamoto H. Effect of alendronate on bone mineral density in spinal cord injury patients: a pilot study. Spinal Cord. 2005;43:341-8.
41. Soleyman-Jahi S, Yousefian A, Maheronnaghsh R, Shokraneh F, Zadegan SA, Soltani A, et al. Evidence-based prevention and treatment of osteoporosis after spinal cord injury: a systematic review. Eur Spine J. 2018;27:1798-814.

42. Zehnder Y, Risi S, Michel D, Knecht H, Perrelet R, Kraenzlin M, et al. Prevention of bone loss in paraplegics over 2 years with alendronate. J Bone Min Res. 2004;19:1067-74.

43. Genant HK, Boonen S, Bolognese MA, Mautalen C, Brown JP, Recknor C, et al. Romosozumab administration is associated with significant improvements in lumbar spine and hip volumetric bone mineral density and content compared with teriparatide. Ann Rheum Dis. 2014;73:172.

44. Bandeira L, Lewiecki EM, Bilezikian JP. Romosozumab for the treatment of osteoporosis. Expert Opin Biol Ther. 2017; $17: 255-63$ 